\title{
Comparison Study of Stem Cell-Derived Extracellular Vesicles for Bone Tissue Engineering
}

\author{
Elham Pishavar ${ }^{1}$, Joshua Copus ${ }^{1}$, Anthony Atala ${ }^{1}$, and Sang Jin Lee ${ }^{1}$ \\ ${ }^{1}$ Wake Forest School of Medicine
}

July 7,2020

\begin{abstract}
Stem cell-derived extracellular vesicles (EVs) have shown great promise in the field of regenerative medicine and tissue engineering. Recently, human bone marrow-derived mesenchymal stem cell (BMSC)-derived EVs have been considered for bone tissue engineering applications. In this study, we evaluated the osteogenic capability of placental stem cell (PSC)-derived EVs and compared them to the well-characterized BMSC-derived EVs. EVs were extracted from three designated time points $(0,7$, and 21 days) after osteogenic differentiation. The results showed that the PSC-derived EVs had much higher protein and lipid concentrations than EVs derived from BMSCs. The extracted EVs were characterized by observing their morphology and size distribution before utilizing next-generation sequencing to determine their miRNA profiles. It was identified that 306 miRNAs within the EVs, of which 64 were significantly expressed in PSC-derived EVs that related to osteogenic differentiation. In vitro osteogenic differentiation study indicated that the late-stage (21-day extracted) derived EVs higher osteogenic enhancing capability when compared to the early-stage derived EVs. We demonstrated that EVs derived from PSCs could be a new source of EVs for bone tissue engineering applications.
\end{abstract}

\section{Introduction}

In the US alone, each year over 500,000 bone grafts are used to treat bone defects or disorders. The bone grafting products and procedures result in a burden of over $\$ 2.5$ billion to the healthcare system, which is expected to double by 2020 [1]. Currently, autologous bone grafts are considered the gold standard for bone regeneration, but they are still limited as they require a second operation to harvest the bone tissue which can often result in donor site morbidity [2]. In order to avoid this, bone tissue engineering is an alternative option that combines scaffolds, cells, and bioactive factors to enhance bone repair and regeneration $[3,4]$.

The use of bone marrow-derived mesenchymal stem cells (BMSCs) has been a common approach for bone tissue engineering applications [5]. To improve the efficacy of stem cells used for bone tissue engineering, bioactive molecules (e.g. growth factors) are commonly incorporated with cells into a scaffolding material. Typically, growth factors such as bone morphogenic proteins (BMPs), vascular endothelial growth factors (VEGFs), and insulin-like growth factors (IGFs) have been used for bone tissue engineering. Especially, BMPs are a family of osteogenic growth factors that include BMP-2 and BMP-7 which have both been incorporated onto FDA approved devices [6]. However, growth factors for bone regeneration have been limited due to their rapid degradation in physiological conditions as well as their deactivation by enzymes [6]. A variety of both organic and inorganic nanoparticles have been utilized to overcome these challenges [7]. Most nanoparticles used for bone tissue engineering have attempted to deliver bioactive molecules such as BMP-2 $[8,9]$ or dexamethasone [10] to directly control differentiation fate of native stem cells or to improve bone mineral deposition, but these osteogenic molecules are still limited in their effectiveness due to their short in vivo half-life, the low loading efficiencies associated with nanoparticles [11], their toxicity and the large quantities of nanoparticles needed for therapeutic effect [6]. 
MicroRNAs (miRNAs) are small, non-coding RNAs that affect cell proliferation, differentiation, and apoptosis by degrading or inhibiting the translation of mRNAs [12]. Unlike other bioactive molecules, miRNAs have the target-specific nature that modulates specific genes [13]. Thus, researchers have recently been interested in delivering miRNAs associated with osteogenesis to improve osteogenic differentiation of BMSCs and therefore bone tissue regeneration [14-16]. Although bone-specific miRNAs play a critical role in bone remodeling [17], their use has been limited due to the difficulty with delivering them to target cells without interference from degrading enzymes. Some research groups have developed lipid-based [18], and even polymeric nanoparticles [19] to deliver miRNAs. Single miRNA delivery still lacks the therapeutic potential of delivering multiple miRNAs especially in bone remodeling where the miRNAs involved act to both inhibit osteoclast activity [20] as well as increasing osteoblast activity [21]. This results in a net increase in the bone mineral deposition that is much greater than the effect of delivering either miRNA alone.

To overcome these limitations, researchers have recently found that naturally derived extracellular vesicles (EVs) can be used as vesicles to deliver multiple miRNAs to target cells. EVs include exosomes, which are small nanovesicles composed of lipid membranes and are excreted by all cell types. These nanovesicles are involved in cell communication, protein transfer, and delivery of microRNAs to surrounding cells [22]. They can be easily extracted from bodily fluids or cell culture medium [22] and also have advantages such as low immunogenicity, high stability, and an intrinsic homing effect which makes them easily absorbed by target cells $[23,24]$, where the osteogenic miRNAs can then be delivered.

BMSC-derived EVs have been shown to play a key role in the maintenance of bone remodeling [25]. These EVs have been shown to enhance the osteogenic differentiation of BMSCs and improve bone formation depending on their miRNA profile. The miRNA profile of a stem cell-derived EV depends on the cell source $[26,27]$ and stage/time point of differentiation. Moreover, the use of BMSCs is limited as they are not readily abundant and require an invasive procedure to be harvested from the donor. The BMSCs are also limited in their passage capabilities, where a typical BMSC will lose its "stemness" around passage 10 [28].

Placental stem cells (PSCs) have a faster proliferation rate $[28,29]$ and can be expanded to larger passage numbers while still maintaining their "stemness." PSCs have been shown to have a proliferation capacity around 30 passages which is almost triple the capacity of adult BMSCs [28]. This is due to the PSCs being closer to embryonic tissue than BMSCs which are typically harvested from adult tissue which gives them limited differentiation capabilities [28]. PSCs can also be easily harvested for autogenic or allogenic use from the postpartum placental tissue without the need for any invasive procedures associated with BMSCs [30]. Taken together, this indicates that PSCs may be an ideal candidate to isolate large quantities of EVs. PSCs have also been shown to exhibit osteogenic differentiation capabilities [31] and are widely used in tissue engineering applications due to their immunomodulation properties [32]. Although EVs from BMSCs are well characterized, the effect of osteogenic differentiation on PSC-derived EV content is poorly understood.

In this study, we aimed to compare the ability of PSC-derived EVs to differentiate with the well-characterized BMSC-derived EVs. We further aim to prove the feasibility of utilizing these EVs for bone tissue engineering applications. Since PSCs exhibit the ability to differentiate into osteogenic cell lines, we hypothesize that EVs isolated from these cells during differentiation could enhance the osteogenic differentiation of BMSCs. We evaluated the effects of cell source and stage of differentiation on the miRNA content of EVs.

\section{Materials and Methods}

\subsection{Cell culture and EV isolation}

Human BMSCs (ATCC, Manassas, VA) were purchased and expanded to passage 3 to 5 before being cultured in EV-free media prepared according to Wang X et al [33]. Human PSCs were obtained and isolated from healthy donors with informed consent approved according to the procedures of the Institutional Review Board (IRB) at Wake Forest University (Winston-Salem, NC), and cells from passage 11 were cultured in EV-free media [33]. Briefly, both BMSCs and PSCs were expanded in growth medium (GM) containing Dulbecco's Modified Eagle's Medium (DMEM, Gibco, Thermo Fisher Scientific, Waltham, MA), 10\% (v/v) 
fetal bovine serum (FBS, ScienCell, Carlsbad, CA), $100 \mathrm{U} / \mathrm{mL}$ penicillin G, and $100 \mathrm{mg} / \mathrm{mL}$ streptomycin (Gibco, Thermo Fisher Scientific) at $37^{\circ} \mathrm{C}$ in an incubator with $5 \% \mathrm{CO}_{2}$ atmosphere and $100 \%$ relative humidity. To induce osteogenic differentiation, BMSCs and PSCs were cultured in osteogenic medium (OM) including $10 \mathrm{nM}$ dexamethasone, $100 \mu \mathrm{M}$ ascorbic acid, and $2 \mathrm{mM} \beta$-glycerophosphate on T175 tissue culture flasks for 21 days. Fresh culture medium was replaced every third day. All chemical reagents were obtained from Millipore Sigma (St. Louis, MO) unless stated otherwise.

For EV isolation, the culture medium was replaced with a serum-free culture medium for $72 \mathrm{~h}$. The media was then collected, and EVs were isolated by a series of differential centrifugation with filtration. Briefly, the supernatant was centrifuged at $1000 \mathrm{~g}$ for $30 \mathrm{~min}$ at $4^{\circ} \mathrm{C}$ to eliminate cellular debris. This was then concentrated and purified with the KR2i TFF system (Spectrum Labs, High Point, NC) using the concentrationdiafiltration-concentration mode. Typically, $500-200 \mathrm{~mL}$ supernatant from the hollow fiber bioreactor was concentrated to approximately $250 \mathrm{~mL}$, diafiltrated with 500-1000 $\mathrm{mL}$ phosphate buffered saline (PBS), and finally concentrated to $10 \mathrm{~mL}$. The hollow fiber filter modules were made from modified polyethersulfone, with a molecular weight cutoff of $500 \mathrm{kDa}$. The flow rate was $80 \mathrm{~mL} / \mathrm{min}$ and the pressure limit was 8 psi for the filter module D02-E500-05-N (used to concentrate supernatant from large volumes to $8 \mathrm{~mL}$ ) [34]. After that supernatant was centrifuged at $500 \mathrm{~g}$ for $20 \mathrm{~min}$ at $4^{\circ} \mathrm{C}$, followed by passing through a $0.22 \mu \mathrm{m}$ filter to eliminate debris. Then, the supernatant was ultracentrifuged at $120,000 \mathrm{~g}$ for $70 \mathrm{~min}$ in a T-647.5 rotor (Sorvall WX Ultra series, Thermo Scientific, USA). The EV pellets were resuspended in PBS and stored at $-80^{\circ} \mathrm{C}$ since it has been previously established that these are optimal long term storage conditions [35].

\subsection{Quantifying EV concentration}

The EV yield was quantified by two different measures as is standard according to the MISEV2018 guidelines. Firstlly, the total protein concentration was measured using the BCA protein assay kit (Thermo Fisher Scientific). Next, total lipid concentration was quantified using a lipid quantification kit (STA-613, Cell Biolabs, San Diego, CA) previously used by other groups investigating EVs [36]. Briefly, $15 \mu \mathrm{L}$ of each EV group were added to a 96 -well plate and incubated uncovered for $30 \mathrm{~min}$ at $90^{\circ} \mathrm{C}$. The samples were then transferred to $4^{\circ} \mathrm{C}$ for 5 min before adding $150 \mu \mathrm{L}$ of $18 \mathrm{M}$ sulfuric acid. They were then incubated at $90^{\circ} \mathrm{C}$ for $10 \mathrm{~min}$ and again transferred to $4^{\circ} \mathrm{C}$ for $5 \mathrm{~min}$. Next, $100 \mu \mathrm{L}$ of each sample was transferred to a clean 96-well plate before being read at $540 \mathrm{~nm}$ to determine the background. $100 \mu \mathrm{L}$ of Vanillin Reagent was then carefully mixed and incubated at $37^{\circ} \mathrm{C}$ for $15 \mathrm{~min}$ before reading at $540 \mathrm{~nm}$ to determine the signal. The background was subtracted from the signal and compared to a previously prepared standard curve.

\subsection{EV characterization}

The EV morphology was observed by transmission electron microscopy (TEM). EVs were fixed with $2 \%$ paraformaldehyde for $15 \mathrm{~min}$; then, the mixture (about $8 \mu \mathrm{L}$ ) was dropped onto carbon-coated copper grids and air-dried for $10 \mathrm{~min}$ before staining with $1 \%$ uranyl acetate two times (6 min each). Images were obtained using an FEI Tecnai BioTwin TEM (Thermo Fisher Scientific). To determine the size distribution, dynamic light scattering (DLS), (Brookhaven Instruments Corporation; Holtsville, NY) was utilized for early (0 day; D0), middle (7 days; D7) and late (21-days; D21) time points of osteogenic differentiation.

\subsection{EV uptake}

To examine whether the BMSCs internalize EVs, the BMSCs were seeded on chamber slides (Millipore, Billerica, MA) at a density of 20,000 cells $/ \mathrm{cm}^{2}$ and cultured overnight. All six EV groups were labeled with a PKH67 Green Fluorescent Cell Linker Kit. PKH67-labeled EVs were diluted in the culture medium and added to the BMSCs in the culture at a concentration of $10 \mu \mathrm{g} / \mathrm{mL}$ media based on reported therapeutic doses of EVs [33]. After $48 \mathrm{~h}$, the cells were washed with PBS to remove non-internalized EVs, and then BMSCs were stained with 4',6-diamidino-2-phenylindole (DAPI) (Thermo Fisher Scientific), which is blue fluorescent nuclei stain. Cells were then fixed with $2 \%$ formaldehyde for $15 \mathrm{~min}$ and washed again. The chamber slides were then mounted with Vectashield HardSet Mounting Medium (Vector Laboratories, Burlingame, CA) and visualized using confocal microscopy (Leica TCS LSI Macro Confocal). 


\subsection{MicroRNA profiling of EVs}

The total miRNA of each EV group was extracted using a miRCURY RNA Isolation Kit (Qiagen, Venlo, Netherlands), according to the manufacturer's protocol. Total RNA including the miRNA fraction was used as the starting material to prepare cDNA libraries using CleanTag ${ }^{\mathrm{TM}}$ Library kit from TriLink Biotechnologies (cat \# L-3206). In brief, adapters are ligated to the 5' phosphate and 3' hydroxyl groups, reverse transcribed, PCR amplified, and then purified using AMPureß XP Beads. Each cDNA Library was validated and checked for size distribution using a High Sensitivity DNA kit on the Agilent 2100 Bioanalyzer. The quantity of each library was measured using the Qubit 3.0 (Thermo Fisher) and loaded on an Illumina Mid Output 150 cycle kit and sequenced using the Illumina NextSeq 500.

Differentially expressed miRNAs, between PSC-EVs and BMSC-EVs, as well as from the early (D0) and late (D21) stages of osteogenic differentiation were further analyzed to predict their target genes and pathways. The target genes of candidate miRNAs were predicted based on two algorithms, DIANA-microT-CDS and DIANA-TarBase v7.0 [37, 38]. The microT threshold was set at a score of 0.8 when microT-CDS algorithms were utilized for target gene prediction. DIANA-mirPath v.3 (http://www.microrna.gr/miRPathv3) [38] was used to perform the hierarchical clustering of miRNAs, and all known KEGG pathways [39] based on their interaction levels using predicted miRNA targets provided by the DIANA-microT-CDS algorithm [40, 41] and/or experimentally validated miRNA interactions derived from DIANA-TarBase v7.0 [42]. The option gene union in the software was selected to merge the results. The graphical output of the program provides an overview of the pathways modulated by selected miRNAs, facilitating the interpretation and presentation of the analysis results. The statistical significance value associated with the identified biological pathways was calculated automatically by the mirPath software, in which Benjamini and Hochberg's false discovery rate (FDR) was applied with the significant threshold set at 0.05 . The FDR is a statistical method for controlling incorrect rejections of the null hypothesis when conducting multiple comparisons which is common during miRNA analysis. This is in accordance with Wang et al. [43] who reported miRNA pathways utilizing the same software systems.

\subsection{EV-stimulated osteogenic differentiation of BMSCs}

BMSCs (passage 3) were seeded at a density of 18,000 cells $/ \mathrm{cm}^{2}$ in 24 -well plates in EV-free OM. After overnight attachment, the culture medium was replaced by media containing EVs. In total, six different EV groups were tested. Both PSC and BMSC EVs isolated at different osteogenic differentiation time points (D0, D7, and D21) were used. The BMSCs were continuously treated with $10 \mu \mathrm{g} / \mathrm{mL} \mathrm{EVs} \mathrm{in} \mathrm{all} \mathrm{in} \mathrm{vitro}$ experiments and media was changed every 3 days.

\subsection{Alkaline phosphatase (ALP) assay}

After 7 and 14 days of treatment, BMSCs were rinsed with DMEM and lysed using Triton X-100 (onestep kit, Thermo Fisher Scientific). The ALP activity was measured using $p$-nitrophenyl phosphate as the substrate. The quantity of $p$-nitrophenol produced was considered directly proportional to the ALP activity according to the protocol.

\subsection{Alizarin Red S staining assay}

To characterize the mineralization of BMSCs after EV treatment an Alizarin Red assay was conducted. Cells were cultured for $7,10,14$, and 21 days with EV treatment before being washed and fixed as in section 2.3. After fixation, the cells were washed with distilled water and incubated with Alizarin Red solution $(\mathrm{pH}$ 4.0-4.3) for $5 \mathrm{~min}$. The extra dye was removed by rinsing with distilled water three times. Samples were observed using light microscopy (Leica DM4000 B). To quantify calcium deposition, $200 \mu \mathrm{L}$ of $10 \%$ acetic 
acid was added to each Alizarin Red stained-well and shaken for 30 min. The cells were then collected and transferred into a microcentrifuge tube where they were vortexed for $30 \mathrm{sec}$. They were then heated to $85^{\circ} \mathrm{C}$ for $10 \mathrm{~min}$ before being cooled on ice for $5 \mathrm{~min}$. The slurry was centrifuged for $15 \mathrm{~min}$ and the supernatant moved to another tube where it was neutralized with $10 \%$ ammonium hydroxide. This was then added to a 96-well plate and the absorbance was read on a plate reader (SpectraMax M5 Microplate Reader). The concentration was compared to a standard curve for quantification. The Alizarin Red staining assay was performed at $7,10,14$, and 21 days.

\subsection{Statistical analysis}

Data are reported three times as the mean \pm standard deviation. The data were analyzed statistically employing a one-way analysis of variance (ANOVA) using GraphPad PRISM@ 8.0 software and $p$-values $<0.05$ indicate statistically significant differences. The miRNA sequencing raw data were normalized based on the DESEQ normalized expression data. KEGG molecular pathway enrichment was reported where FDR was less than 0.001 .

\section{Results}

\subsection{Characterization of stem cell-derived EVs}

EVs were isolated from both BMSCs and PSCs cultured in growth media (D0) as well as osteogenic media at 7 days (D7) and 21 days (D21) of culture. The yield of the BMSC-derived EVs was 71.0 $\pm 38.3,149.3 \pm 22.9$, and $123.6 \pm 16.9 \mu \mathrm{g} / \mathrm{mL}$ for D0, D7, and D21, respectively. BMSCs exhibited similar yields to the values reported in the literature [44], but PSCs consistently yielded higher EV protein quantities in comparison to BMSCs. The PSC-derived EVs had yields of $394.6 \pm 38.2,959.3 \pm 59.7$, and $695.7 \pm 90.8 \mu \mathrm{g} / \mathrm{mL}$ at D0, D7, and D21, respectively (Figure 2A ). The lipid concentration of the EVs was also quantified so that a comparison could be made between the protein to lipid ratios of each EV group as is standard according to ISEV to ensure EVs are pure and the isolation method does not co-isolate unbound proteins. The BMSC-derived EVs had lipid concentrations of $185.2 \pm 17.2,495.2 \pm 32.9$, and $1339.37 \pm 74.2 \mu \mathrm{g} / \mathrm{mL}$ at D0, D7, and D21 respectively. The PSC-derived EVs had lipid concentrations of $3486.5 \pm 227.1,818.1 \pm 85.8$, and $247.7 \pm 8.2 \mu \mathrm{g} / \mathrm{mL}$ at D0, D7, and D21 respectively (Figure 2B ). This means that for the protein to lipid ratios, BMSC-derived EVs had a ratio of $0.383,0.302$, and 0.092 at D0, D7, and D21 respectively, while the PSC-derived EVs were $0.113,1.172$, and 2.808 at $\mathrm{D} 0, \mathrm{D} 7$, and D21, respectively.

EVs were characterized by TEM and DLS to confirm that the size and morphology were consistent with the values and shape reported by other groups isolating EVs. The TEM images showed that all six groups of examined EVs had a cup-shaped morphology and an average hydrodynamic diameter between 120-150 nm (Figure 2C) . The TEM images revealed that the different groups of isolated EVs featured similar shape and size ranges, confirming that the techniques for isolation yielded consistent batches of EVs.

DLS was also performed to quantitatively confirm the EV size(Figure 2D). DLS is able to calculate the hydrodynamic diameter of EVs from the Stokes-Einstein Equation, which is used to characterize how large particles move slower due to Brownian motion. From this experiment, we found that the BMSC-derived EVs had diameters of $137.4 \pm 11.97,158.7 \pm 29.41$, and $218.3 \pm 20.3 \mathrm{~nm}$ on D0, D7, and D21, respectively. The PSC-derived EVs had diameters of $135.5 \pm 1.17,129.3 \pm 25.22$, and $197.5 \pm 34.85 \mathrm{~nm}$ on D0, D7, and D21, respectively.

\subsection{Internalization of EVs by BMSCs}

To explore whether EVs could enter the cytoplasm of BMSCs, the six groups of EVs were labeled with green fluorescence dye PKH67 and incubated with BMSCs for $48 \mathrm{~h}$. Fluorescence microscopy analysis showed that the BMSCs were capable of internalizing the EVs of all groups(Figure 3) . The internalized PKH67 fluorescence varied very little between EV groups and across cells within the culture, which suggests that the EVs were homogenously internalized.

\subsection{MicroRNA profiles of EVs during expansion and differentiation}


Among the variety of molecules that are contained in EVs, miRNAs have attracted the most attention because of their regulatory roles in gene expression [45]. For this reason, we determined the content of miRNAs within our EVs. We then moved onto next-generation sequencing to determine the miRNA profiles contained within our EVs. A total of 306 miRNAs were identified in all samples (Table S1) . Clustering analysis of the miRNA expression in the six groups of EVs revealed marked differences in miRNA expression, and the software system was able to determine biological pathways that were significantly impacted by the identified miRNAs (Figure 4A and Table S2). Among the differentially expressed miRNAs in D0 versus D21 (Table S3), miR-186, miR-210, miR-181c-5p, and miR-146a-5p were increased two-fold and miR-133 and miR-485 were decreased two-fold in the last stage. Meanwhile, miR-10, miR-27a, miR-192 increased which have been reported as late markers of osteogenesis, and miR-31 decreased. In addition, miR-10 and miR-138 were expressed six-fold higher in BMSCs versus PSCs(Figure 4B, C). The top 34 KEGG pathways were also reported which indicated the potential upregulated target genes of the 301 miRNAs that were identified in both BMSC and PSC EVs (Figure S1). Of the potential KEGG pathways analyzed Wnt signaling was of particular interest due to its role in osteogenic differentiation of MSCs [46].

\subsection{EV-stimulated osteogenic differentiation of BMSCs}

EVs derived from PSCs and BMSCs during different stages of differentiation were applied as stimuli to treat undifferentiated BMSCs. After 7 and 14 days of treatment, the ALP activity was measured as an early indicator of cell differentiation towards the osteogenic lineage(Figure 5). Both BMSC-D21 and PSC-D21 EVs significantly increased ALP activity at 7 days of culture in comparison to the positive control in OM. At 14 days, almost all EV groups showed a significant increase in ALP activity, except for BMSC-D0.Alizarin Red S staining (Figure 6A) and quantification assay(Figure 6B) demonstrated that calcium deposition significantly increased after 14-day induction by all EVs in comparison to the positive control in OM.

\section{Discussion}

The therapeutic potential of BMSC-derived EVs has been well documented [47-50]. Their role in bone remodeling has been investigated bothin vitro and in vivo, and it has been shown that many of the key factors for regulating bone remodeling are targeted by miRNAs contained within the EVs [51, 52]. Researchers have focused mainly on utilizing these EVs and their biologically active content to address pathological bone diseases such as osteoporosis, but these EVs also exhibit the potential to improve stem cell differentiation within tissue-engineered bone scaffolds due to their ability to accelerate bone mineral deposition. Most studies utilize EVs derived from BMSCs or mineralizing osteoblasts to achieve this effect, so there exists a lack of knowledge on utilizing alternate cell sources to harvest EVs with the potential to improve bone mineral formation [53].

In this study, we compared EVs derived from BMSCs and PSCs during the process of osteogenic differentiation in an attempt to better understand the role that cell source and culture conditions have on the therapeutic effect of these EVs. It has been shown that the miRNA profile within EVs changes depending on the stage of differentiation [54]; however, this effect has not been studied while comparing different mesenchymal stem cells. We isolated and characterized EVs derived from BMSCs and PSCs, and investigated the differentiation capabilities of EVs secreted under different stages of osteogenic differentiation. Within this study, we aimed to determine the effects of both cell type and differentiation stage, on EV content, and the downstream effect on the regulation of osteogenic differentiation [54]. By doing so, we have proven the feasibility of utilizing alternative cell sources of EVs for future studies involving osteogenic differentiation.

The isolated EV groups did not exhibit any statistically significant differences in size, regardless of cell source. This confirms the consistency of the isolation process utilized for EV separation. When comparing between differentiation time within the same cell source, the isolated EVs exhibited larger size distributions at D21 compared to D0 with $p=0.004$ for BMSC-derived and $p=0.0369$ for PSC-derived EVs. This may be due to the larger cell membrane surface area available for EV formation since the cell morphology of MSCs become less spindle-like due to the cells differentiating into osteoblast-like cells [55].

Characterizing the protein concentration between cell groups yielded large differences between cell source 
with the PSC-derived EVs consistently yielding higher protein concentrations. When analyzing the lipid concentrations of each EV group, we found that the PSC-derived EVs also had significantly higher lipid concentrations at all time points with the exception of D21. The trend of isolating both higher protein concentrations and lipid concentrations from PSCs suggests that there was a larger total yield of EVs from these cells when compared to BMSCs. When comparing the protein to lipid ratio, which is a common quality assurance step to ensure that the isolation method is not co-isolating unwanted proteins, the PSC-derived EVs had slightly higher protein to lipid ratios than BMSCs. All groups fell within an acceptable range of a protein to lipid ratio $<5$, which was previously described by Osteikoetxea et al [56]. Another interesting result is that as the time of differentiation increased, the PSCs yielded lower lipid concentrations while the lipid concentration increased for BMSCs. Taken together with the in vitro data, which dosages were based off of protein concentrations, this implies that the PSC-derived EVs required a lower total number of EVs to achieve the same targeted differentiation effect as the BMSC-derived EVs.

The ability of PSC-derived EVs to be internalized by BMSCs was an important aspect of this study, suggesting that the EVs are not internalized by a cell-specific pathway. It was beyond the focus of this study to identify the individual surface proteins and glycoproteins expressed on each EV group or to determine the efficiency at which these EVs were internalized. But, by fluorescently labeling the EV membrane it was observed that the EVs were internalized by cells in vitroregardless of cell source or differentiation time. Taken in combination with the results of the differentiation experiments, it can be assumed that EVs were internalized efficiently enough to achieve the targeted effect.

It was outside this study's scope to experimentally confirm the effect of each miRNA identified within our EVs. We observed a trend within both PSC and BMSC-derived EVs that resulted in the improved mineral deposition in $2 \mathrm{D}$ in vitro culture when delivering EVs from cells that were exposed to longer osteogenic differentiation times. This hints that the trend for miRNA profiles observed in BMSCs between stages of differentiation is consistent within PSCs as well [54]. It is unclear whether these miRNAs are solely responsible for the osteogenic differentiation effect identified in the D21 group of EVs. It is much more likely that the effects observed are a result of a combination of bioactive molecules contained within the EVs, including these miRNAs. Regardless, we identified the relative expression levels of over 300 miRNAs and analyzed the differences in expression between cell source and stage of differentiation in an attempt to gain insight on whether these EVs are comparable in terms of miRNA profile.

Among the the miRNAs differentially expressed between PSCs and BMSCs, miR-10b was dramatically increased in EVs from the D21 BMSC-derived EVs and was about 123 folds higher than PSCs (Table S1 ). The TargetScan 7.2 software, which predicts biological targets of miRNAs, precited that miR-10b can affect ID4 and indirectly promote osteoblast differentiation by enhancing RunX2 transcriptional activity [57]. Furthermore, it has been reported that miR-10b promotes the migration of MSCs into the bone microenvironment $[33,58]$. It was also determined that the expression level of miR-21 increased 2 fold higher late-stage PSC and BMSC EVs in comparison to D0 EVs (Table S1). The upregulation of miR-21 promotes osteogenesis through the PI3K/AKT/B-actin pathway and has effects on the mRNA expression RUNX2, ALK, and OCN [59]. MiR-126-5p has also been found to regulate osteoclast differentiation and plays a role in bone remodeling through the inhibition of MMP-13 [60]. Pathway analysis revealed the differentially expressed miRNAs targeted genes through multiple signaling pathways such as Wnt signaling (Figure S1), MAPkinase, and TGF $\beta$ pathways (Table S2). The varied, non-consistent miRNA expressions between cell type and differentiation stage suggest a much more general trend towards osteogenesis, through many different pathways, as opposed to the expression of specific osteo-miRNAs that affect individual genes or pathways.

There were 3 miRNAs associated with increased osteogenesis that were expressed significantly higher in late-stage PSCs when compared to BMSCs. Most were associated with promoting osteoblast differentiation such as miR-146, miR-515, and miR-520a (Table S3). The PSCs also significantly expressed two miRNAs, miR-512 and miR516b that were associated with inhibiting osteogenic differentiation and downregulating osteoblast differentiation, respectively. There were also various other osteo-related miRNAs that were downregulated at the later stages of differentiation such as miR-133 which is associated with the inhi- 
bition of osteoblast differentiation [61]. Although its expression decreased over time, it was still expressed approximately 2-fold higher at late-stage PSC EV in comparison to BMSC EV. Other negative regulators of osteogenesis, such as let-7i, were found to be 6-fold higher in BMSC EVs than PMSC EVs while still maintaining the trend of decreasing expression at later time points of differentiation. The importance of the expression levels of individual miRNAs remains unclear until further studies can identify their specific functions. When analyzing the trends of osteo-related miRNA, these miRNA expression levels are still consistent with the general trend towards increased osteogenesis in the late-stage EVs regardless of cell source. This was confirmed within vitro uptake and differentiation experiments.

Based on our in vitro experiments, EVs derived from late-stage differentiation were able to achieve faster differentiation rates and improved mineral deposition when compared to our control. This is the result of delivering osteogenic miRNA as well as other potential osteogenic factors contained within the vesicles. Although the cell source had a large impact on the variety of miRNA contained within the EVs, the overall osteogenic impact was similar between the groups when comparing EVs derived at the same stage. All of the in vitroexperiments chosen have been previously used to confirm osteogenic differentiation [62]. The improved bone mineral deposition resulting from delivering late-stage PSC and BMSC-derived EVs could potentially lead to the development of enhanced bone tissue engineering scaffolds or even improve fracture or non-union healing.

All of this data taken together signifies a new candidate from which to isolate osteogenic EVs. PSC's ease of donor availability, fast proliferation time, and high EV potency improve the likelihood that they will make a suitable candidate for clinical translation. The ability to easily scale up the isolation methods for these EVs, combined with their enhanced osteogenic differentiation rates, demonstrate their viability for future studies involving bone tissue engineering.

\section{Conclusions}

We compared PSC-derived EV's ability to differentiate cells in vitro with the well-characterized BMSCderived EVs and proved the feasibility of utilizing these EVs for bone tissue engineering. We found that ALP activity and calcium deposition of BMSCs significantly increased when treated with late-stage derived EVs, regardless of cell source. We have presented a comparative analysis of the miRNA content of these EVs, as well as identified potential pathways upon which these miRNAs are acting upon the cells. Since PSCs grow at a significantly faster rate than BMSCs, and also yield more potent EVs, they are a promising alternative source to utilizing BMSC-derived EVs for studies that require large amounts of EVs or need a high yield for therapeutic effects. During differentiation, EVs derived from late-stage osteogenic culture enhanced the rate of differentiation and mineralization of BMSCs regardless of stem cell source. This is a result of the uptake and delivery of the biologically active molecules contained within the EVs such as miRNAs. It is our hope that this information can lead to future studies improving the delivery mechanisms of EVs so they can overcome limitations with current bone grafting techniques by increasing native bone cell activity.

\section{Acknowledgments}

This study was supported by the National Science Foundation (NSF, Award \#1663128) and the State of North Carolina. This work was supported, in part, by the Cancer Genomics Shared Resource supported by the Wake Forest Baptist Comprehensive Cancer Center's NCI Cancer Center Support Grant P30CA01219739.

\section{References}

[1] Amini, A. R., Laurencin, C. T., Nukavarapu, S. P., Bone tissue engineering: recent advances and challenges. Critical Reviews in Biomedical Engineering 2012, 40 .

[2] Brooks, P. M., The burden of musculoskeletal disease - a global perspective. Clinical rheumatology 2006, $25,778-781$. 
[3] O'Keefe, R. J., Mao, J., Bone tissue engineering and regeneration: from discovery to the clinic - an overview. Tissue engineering part B: reviews 2011, 17 , 389-392.

[4] Holland, T. A., Mikos, A. G., Biodegradable polymeric scaffolds. Improvements in bone tissue engineering through controlled drug delivery. Adv Biochem Eng Biotechnol 2006, 102 , 161-185.

[5] Marolt Presen, D., Traweger, A., Gimona, M., Redl, H., Mesenchymal Stromal Cell-Based Bone Regeneration Therapies: From Cell Transplantation and Tissue Engineering to Therapeutic Secretomes and Extracellular Vesicles. Front Bioeng Biotechnol 2019, 7, 352.

[6] De Witte, T.-M., Fratila-Apachitei, L. E., Zadpoor, A. A., Peppas, N. A., Bone tissue engineering via growth factor delivery: from scaffolds to complex matrices. Regenerative biomaterials 2018,5 , 197-211.

[7] Vieira, S., Vial, S., Reis, R. L., Oliveira, J. M., Nanoparticles for bone tissue engineering. Biotechnology progress2017, 33 , 590-611.

[8] Bessa, P. C., Machado, R., Nürnberger, S., Dopler, D., et al. , Thermoresponsive self-assembled elastinbased nanoparticles for delivery of BMPs. Journal of Controlled Release 2010, 142 , 312-318.

[9] Cao, L., Wang, J., Hou, J., Xing, W., Liu, C., Vascularization and bone regeneration in a critical sized defect using 2-N, 6-O-sulfated chitosan nanoparticles incorporating BMP-2. Biomaterials 2014,35 , 684-698.

[10] Li, L., Zhou, G., Wang, Y., Yang, G., et al. , Controlled dual delivery of BMP-2 and dexamethasone by nanoparticle-embedded electrospun nanofibers for the efficient repair of critical-sized rat calvarial defect. Biomaterials 2015, 37, 218-229.

[11] Zhang, S., Wang, G., Lin, X., Chatzinikolaidou, M., et al. , Polyethylenimine-coated albumin nanoparticles for BMP-2 delivery.Biotechnology progress 2008, 24 , 945-956.

[12] Wang, J., Liu, S., Li, J., Zhao, S., Yi, Z., Roles for miRNAs in osteogenic differentiation of bone marrow mesenchymal stem cells.Stem cell research \& therapy 2019, 10 , 197.

[13] Kim, N., Yoo, J. J., Atala, A., Lee, S. J., Combination of small RNAs for skeletal muscle regeneration. FASEB J 2016,30 , 1198-1206.

[14] Dong, S., Yang, B., Guo, H., Kang, F., MicroRNAs regulate osteogenesis and chondrogenesis. Biochemical and biophysical research communications 2012, 418, 587-591.

[15] Li, Y., Fan, L., Liu, S., Liu, W., et al. , The promotion of bone regeneration through positive regulation of angiogenic-osteogenic coupling using microRNA-26a. Biomaterials2013, 34, 5048-5058.

[16] Qin, Y., Wang, L., Gao, Z., Chen, G., Zhang, C., Bone marrow stromal/stem cell-derived extracellular vesicles regulate osteoblast activity and differentiation in vitro and promote bone regeneration in vivo. Scientific reports 2016, 6 , 21961.

[17] Jing, D., Hao, J., Shen, Y., Tang, G., et al. , The role of microRNAs in bone remodeling. International journal of oral science 2015, 7, 131 .

[18] Zhang, Y., Wang, Z., Gemeinhart, R. A., Progress in microRNA delivery. Journal of controlled release 2013, 172, 962-974.

[19] Radmanesh, F., Abandansari, H. S., Pahlavan, S., Alikhani, M., et al. , Optimization of miRNA delivery by using a polymeric conjugate based on deoxycholic acid-modified polyethylenimine.International journal of pharmaceutics 2019, $565,391-408$.

[20] Shi, C., Qi, J., Huang, P., Jiang, M., et al. , MicroRNA-17/20a inhibits glucocorticoid-induced osteoclast differentiation and function through targeting RANKL expression in osteoblast cells. Bone 2014, 68, 67-75.

[21] Tang, X., Lin, J., Wang, G., Lu, J., MicroRNA-433-3p promotes osteoblast differentiation through targeting DKK1 expression. PloS one 2017, 12 , e0179860. 
[22] Garcia-Contreras, M., Shah, S. H., Tamayo, A., Robbins, P. D., et al. , Plasma-derived exosome characterization reveals a distinct microRNA signature in long duration Type 1 diabetes.Scientific reports $2017,7,5998$.

[23] Li, W., Liu, Y., Zhang, P., Tang, Y., et al. , Tissue-engineered bone immobilized with human adipose stem cells-derived exosomes promotes bone regeneration. ACS applied materials \& interfaces 2018, 10 , $5240-5254$.

[24] Andaloussi, S. E. L., Lakhal, S., Mager, I., Wood, M. J. A., Exosomes for targeted siRNA delivery across biological barriers. Advanced drug delivery reviews 2013, 65 , 391-397.

[25] Behera, J., Tyagi, N., Exosomes: mediators of bone diseases, protection, and therapeutics potential. Oncoscience 2018,5, 181.

[26] Barlow, S., Brooke, G., Chatterjee, K., Price, G., et al. , Comparison of human placenta-and bone marrow-derived multipotent mesenchymal stem cells. Stem cells and development 2008,17, 1095-1108.

[27] Huang, L., Ying, H., Chen, Z., long Zhu, Y., et al. , Down-regulation of DKK1 and Wnt1/ß-catenin pathway by increased homeobox B7 resulted in cell differentiation suppression of fetal intrauterine growth retardation in human placenta. Placenta 2019.

[28] Sung, H. J., Hong, S. C., Yoo, J. H., Oh, J. H., et al. , Stemness evaluation of mesenchymal stem cells from placentas according to developmental stage: comparison to those from adult bone marrow. Journal of Korean medical science 2010, 25 , 1418-1426.

[29] in't Anker, P. S., Scherjon, S. A., Kleijburg-van der Keur, C., de Groot-Swings, G. M. J. S., et al. , Isolation of mesenchymal stem cells of fetal or maternal origin from human placenta. Stem cells 2004, 22, 1338-1345.

[30] Zhang, Y., Li, C.-d., Jiang, X.-x., Li, H.-l., et al. , Comparison of mesenchymal stem cells from human placenta and bone marrow. Chin Med $J$ (Engl) 2004, 117, 882-887.

[31] Youssef, A., Han, V. K. M., Regulation of osteogenic differentiation of placental-derived mesenchymal stem cells by insulin-like growth factors and low oxygen tension. Stem cells international 2017, 2017 .

[32] Wolbank, S., Peterbauer, A., Fahrner, M., Hennerbichler, S., et al. , Dose-dependent immunomodulatory effect of human stem cells from amniotic membrane: a comparison with human mesenchymal stem cells from adipose tissue. Tissue engineering 2007, 13 , 1173-1183.

[33] Wang, X., Omar, O., Vazirisani, F., Thomsen, P., Ekström, K., Mesenchymal stem cell-derived exosomes have altered microRNA profiles and induce osteogenic differentiation depending on the stage of differentiation. PloS one 2018, 13 , e0193059.

[34] Yoo, K. W., Li, N., Makani, V., Singh, R. N., et al. , Large-scale preparation of extracellular vesicles enriched with specific microRNA. Tissue Engineering Part C: Methods 2018, 24, 637-644.

[35] Lee, M., Ban, J.-J., Im, W., Kim, M., Influence of storage condition on exosome recovery. Biotechnology and bioprocess engineering 2016, 21 , 299-304.

[36] Théry, C., Witwer, K. W., Aikawa, E., Alcaraz, M. J., et al. , Minimal information for studies of extracellular vesicles 2018 (MISEV2018): a position statement of the International Society for Extracellular Vesicles and update of the MISEV2014 guidelines. Journal of Extracellular Vesicles 2018, 7 , 1535750.

[37] Vlachos, I. S., Zagganas, K., Paraskevopoulou, M. D., Georgakilas, G., et al. , DIANA-miRPath v3. 0: deciphering microRNA function with experimental support. Nucleic acids research 2015, 43, W460-W466.

[38] Vlachos, I. S., Hatzigeorgiou, A. G., Functional analysis of miRNAs using the DIANA Tools online suite, Drug Target miRNA, Springer 2017, pp. 25-50. 
[39] Maragkakis, M., Reczko, M., Simossis, V. A., Alexiou, P., et al. , DIANA-microT web server: elucidating microRNA functions through target prediction. Nucleic Acids Research 2009, 37, W273-W276.

[40] Paraskevopoulou, M. D., Georgakilas, G., Kostoulas, N., Vlachos, I. S., et al. , DIANA-microT web server v5. 0: service integration into miRNA functional analysis workflows. Nucleic acids research 2013, 41 , W169-W173.

[41] Reczko, M., Maragkakis, M., Alexiou, P., Grosse, I., Hatzigeorgiou, A. G., Functional microRNA targets in protein coding sequences. Bioinformatics 2012, 28 , 771-776.

[42] Vlachos, I. S., Paraskevopoulou, M. D., Karagkouni, D., Georgakilas, G., et al. , DIANA-TarBase v7. 0: indexing more than half a million experimentally supported miRNA: mRNA interactions. Nucleic acids research 2014, 43 , D153-D159.

[43] Wang, X., Liao, X., Huang, K., Zeng, X., et al. , Clustered microRNAs hsa-miR-221-3p/hsa-miR-222$3 \mathrm{p}$ and their targeted genes might be prognostic predictors for hepatocellular carcinoma.Journal of Cancer 2019, $10,2520-2533$.

[44] Tang, Y.-T., Huang, Y.-Y., Zheng, L., Qin, S.-H., et al. , Comparison of isolation methods of exosomes and exosomal RNA from cell culture medium and serum. International journal of molecular medicine 2017, $40,834-844$.

[45] Zhang, J., Li, S., Li, L., Li, M., et al. , Exosome and exosomal microRNA: trafficking, sorting, and function. Genomics, proteomics $\& 3$ bioinformatics 2015, 13 , 17-24.

[46] Kim, J. H., Liu, X., Wang, J., Chen, X., et al. , Wnt signaling in bone formation and its therapeutic potential for bone diseases. Therapeutic advances in musculoskeletal disease 2013,5 , 13-31.

[47] Hao, Z. C., Lu, J., Wang, S. Z., Wu, H., et al. , Stem cell-derived exosomes: A promising strategy for fracture healing. Cell Proliferation 2017, 50 , e12359.

[48] Fang, S., Li, Y., Chen, P., Osteogenic effect of bone marrow mesenchymal stem cell-derived exosomes on steroid-induced osteonecrosis of the femoral head. Drug design, development and therapy 2019,13 , 45.

[49] Liu, J., Li, D., Wu, X., Dang, L., et al. , Bone-derived exosomes. Current opinion in pharmacology 2017, $34,64-69$.

[50] Liu, M., Sun, Y., Zhang, Q., Emerging role of extracellular vesicles in bone remodeling. Journal of dental research 2018,97, 859-868.

[51] Qin, Y., Sun, R., Wu, C., Wang, L., Zhang, C., Exosome: a novel approach to stimulate bone regeneration through regulation of osteogenesis and angiogenesis. International journal of molecular sciences 2016, 17, 712 .

[52] Qin, Y., Wang, L., Gao, Z., Chen, G., Zhang, C., Bone marrow stromal/stem cell-derived extracellular vesicles regulate osteoblast activity and differentiation in vitro and promote bone regeneration in vivo. Scientific reports 2016, 6 , 1-11.

[53] Cui, Y., Luan, J., Li, H., Zhou, X., Han, J., Exosomes derived from mineralizing osteoblasts promote ST2 cell osteogenic differentiation by alteration of microRNA expression. FEBS letters 2016, 590 , 185-192.

[54] Gao, J., Yang, T., Han, J., Yan, K., et al. , MicroRNA expression during osteogenic differentiation of human multipotent mesenchymal stromal cells from bone marrow. Journal of cellular biochemistry 2011, $112,1844-1856$.

[55] Kalajzic, I., Matthews, B. G., Torreggiani, E., Harris, M. A., et al. , In vitro and in vivo approaches to study osteocyte biology. Bone 2013, 54, 296-306.

[56] Osteikoetxea, X., Balogh, A., Szabo-Taylor, K., Nemeth, A., et al. , Improved characterization of EV preparations based on protein to lipid ratio and lipid properties. PloS one 2015,10 , e0121184. 
[57] Tokuzawa, Y., Yagi, K., Yamashita, Y., Nakachi, Y., et al. , Id4, a new candidate gene for senile osteoporosis, acts as a molecular switch promoting osteoblast differentiation. PLoS genetics 2010, 6 , e1001019.

[58] Weilner, S., Skalicky, S., Salzer, B., Keider, V., et al. , Differentially circulating miRNAs after recent osteoporotic fractures can influence osteogenic differentiation. Bone 2015,79, 43-51.

[59] Meng, Y. B., Li, X., Li, Z. Y., Zhao, J., et al. , microRNA-21 promotes osteogenic differentiation of mesenchymal stem cells by the PI3K/ß-catenin pathway. Journal of Orthopaedic Research 2015, 33 , 957-964.

[60] Wu, Z., Yin, H., Liu, T., Yan, W., et al. , MiR-126-5p regulates osteoclast differentiation and bone resorption in giant cell tumor through inhibition of MMP-13. Biochemical and biophysical research communications 2014, 443, 944-949.

[61] Lv, H., Sun, Y., Zhang, Y., MiR-133 is involved in estrogen deficiency-induced osteoporosis through modulating osteogenic differentiation of mesenchymal stem cells. Medical science monitor: international medical journal of experimental and clinical research 2015, 21, 1527.

[62] Jaiswal, N., Haynesworth, S. E., Caplan, A. I., Bruder, S. P., Osteogenic differentiation of purified, culture-expanded human mesenchymal stem cells in vitro. Journal of cellular biochemistry1997, 64 , 295312.

\section{Figure Captions}

Figure 1. Schematic diagram of the experimental design for comparison study.

Figure 2. Characterization of stem cell-derived EVs: (A) Protein concentrations. (B) Lipid concentrations. ( C) Morphological analysis by TEM of EVs derived from PSCs and BMSCs at D0, D7, and D21. (D) DLS analysis of EVs show a similar hydrodynamic diameter around 40-100 $\mathrm{nm}$.

Figure 3. Visualization of EVs in BMSCs. EVs are labeled in green with PKH67 while cell nuclei was stained blue with DAPI.

Figure 4. (A) Altered miRNA profiles of EVs derived from 2 type stem cells and between D0 and D21. (B) Pie chart showing the correlation of the top 44 abundant microRNAs (based on FDR $<=0.05$ ) among EVs derived from both cell types. (C) Heatmap of the most abundant miRNAs depiction expression change between cell types and over time.

Figure 5. ALP activity of BMSCs after incubation with EVs $(10 \mu \mathrm{g} / \mathrm{ml})$ after (A) 7 and (B) 14 days of osteogenic differentiation.

Figure 6. Calcium content assay: (A) Alizarin Red S stained images at 7, 10, and 14 days after osteogenic differenation. (B) Alizarin Rdd quantification at 10, 14, and 21 days after osteogenic differenation.

\section{Hosted file}

image1.emf available at https://authorea.com/users/340074/articles/467191-comparison-studyof-stem-cell-derived-extracellular-vesicles-for-bone-tissue-engineering

\section{Figure 1.}

\section{Hosted file}

image2.emf available at https://authorea.com/users/340074/articles/467191-comparison-studyof-stem-cell-derived-extracellular-vesicles-for-bone-tissue-engineering

Figure 2.

\section{Hosted file}


image3.emf available at https://authorea.com/users/340074/articles/467191-comparison-studyof-stem-cell-derived-extracellular-vesicles-for-bone-tissue-engineering

\section{Figure 3.}

\section{Hosted file}

image4.emf available at https://authorea.com/users/340074/articles/467191-comparison-studyof-stem-cell-derived-extracellular-vesicles-for-bone-tissue-engineering

\section{Figure 4.}

\section{Hosted file}

image5.emf available at https://authorea.com/users/340074/articles/467191-comparison-studyof-stem-cell-derived-extracellular-vesicles-for-bone-tissue-engineering

\section{Figure 5.}

\section{Hosted file}

image6.emf available at https://authorea.com/users/340074/articles/467191-comparison-studyof-stem-cell-derived-extracellular-vesicles-for-bone-tissue-engineering

\section{Figure 6.}

\section{TOC}

\section{Hosted file}

image7.emf available at https://authorea.com/users/340074/articles/467191-comparison-studyof-stem-cell-derived-extracellular-vesicles-for-bone-tissue-engineering 